Service social

\title{
Méthodologie de l'intervention sociale et interculturalité
}

\section{Guy Bilodeau}

Volume 42, numéro 1, 1993

\section{Culture et intervention}

URI : https://id.erudit.org/iderudit/706598ar

DOI : https://doi.org/10.7202/706598ar

Aller au sommaire du numéro

\section{Éditeur(s)}

École de service social de l'Université Laval

ISSN

1708-1734 (numérique)

Découvrir la revue

Citer cet article

Bilodeau, G. (1993). Méthodologie de l'intervention sociale et interculturalité. Service social, 42(1), 25-48. https://doi.org/10.7202/706598ar

\section{Résumé de l'article}

Cet écrit fait état des réflexions et des travaux d'un groupe de praticiens qui, à partir d'études de cas, ont pu dégager un ensemble de principes, de valeurs, d'attitudes et de comportements professionnels qui devraient plus particulièrement être pris en considération dans l'intervention interculturelle. Ces réflexions ont été placées dans le cadre théorique de la méthodologie du travail social.

C'est dans cette densité de réalités multiples que le travailleur social, sollicité par le client ou mandaté par l'organisation sociale, devra en toute maîtrise de sa méthodologie offrir un soutien qui permettra à la personne immigrante (groupe ou communauté) de rehausser sa compétence sociale dans le nouveau tissu social qu'elle doit se constituer du fait de la situation de transition que l'immigration lui impose. 


\section{Méthodologie de l'intervention sociale et interculturalité}

Guy BILODEAU

Travailleur social et professeur agrégé École de service social

Université Laval

avec la collaboration des professionnels du Centre de services sociaux du Montréal métropolitain

Marguerite BÉLANGER, travailleuse sociale José DA COSTA, psychologue Lise GRÉGOIRE, bachelière en droit Michèle LAVOIE-CHIASSON, anthropologue Jean-Paul MAILLOUX, psychologue

Nicole MILLETTE, travailleuse sociale

Berthe SCOTT-LACHAMBRE, techn. en assistance sociale Marie NALTCHAYAN, travailleuse sociale

\section{INTRODUCTION}

Depuis quelques années l'évolution de la société québécoise et les changements importants du tissu social au Québec ont suscité l'émergence d'une préoccupation généralisée: celle de tracer les nouveaux contours d'une réalité quotidiennement vécue «au pluriel ». En effet, d'une relative homogénéité faite de valeurs convergentes, le Québec est passé à l'ère du métissage et d'une pluralité de valeurs gros de richesse, certes, mais dont le potentiel ne masque pas les chocs qu'il est convenu d'appeler culturels... 
$C^{\prime}$ est dans ce contexte que la profession de travail social est remise en question : la profession est-elle adaptée à sa mission aujourd'hui ? D'aucuns soutiennent qu'il n'y a pas d' «approche» spécifiquement reliée à la situation d'interculturalité. D'autres mettent en relief les obstacles rencontrés en situation de choc culturel et en ont pour preuve l'inaccessibilité des services du réseau aux communautés culturelles. Placés dans des situations où une dimension interculturelle heurte de front leur savoir-faire professionnel, les intervenants s'interrogent sur leurs compétences. $Y$ aurait-il une façon de faire, une méthode spécialement adaptée aux situations où les intervenants sont en interculturalité? Devrait-on construire des grilles d'évaluation spécifiques? Le travail en situation d'interculturalité a-t-il une parenté avec le processus d'intervention développé dans les différentes approches en service social? Ou encore peut-on repérer dans la méthodologie d'intervention les dimensions où il deviendrait impératif de pratiquer une vigilance particulière en situation d'interculturalité?

Le présent texte, d'une part, fait état des réflexions ${ }^{1}$ et des travaux $d^{\prime} u n$ groupe de praticiens du Centre des services sociaux du Montréal métropolitain qui, à partir d'études de cas, ont pu dégager un ensemble de principes, de valeurs, d'attitudes et de comportements professionnels qui devraient plus particulièrement être pris en considération dans l'intervention interculturelle (Naltchayan, 1991). D'autre part, ces réflexions - cette sagesse professionnelle - ont été placées dans le cadre théorique de la méthodologie du travail social.

Ce document se présente comme un guide à l'intention d'intervenants qui désirent travailler dans le domaine de l'interculturalité, guide qui allie pratique et cadre théorique et qui propose de revoir les parcours méthodologiques de l'intervention sociale en pointant les éléments propres à l'interculturalité qui sont à considérer.

\section{MÉTHODOLOGIE DE L'INTERVENTION SOCIALE}

L'acte professionnel du travailleur social a pour objet le fonctionnement social, c'est-à-dire les interactions des individus, des familles, des groupes et des collectivités avec leur environnement, dans un but réciproque de développement humain et social (Corporation professionnelle des travailleurs sociaux, 1991). L'intervention du travailleur social dans les interactions personne-environnement se produit dès lors que la personne ou des membres de l'environnement jugent qu'un problème ne se règle plus de lui-même

1. Ces réflexions sont insérées dans ce texte en italique. 
ou qu'une situation est devenue intolérable. Jusque-là, la personne et l'environnement avaient trouvé en eux les possibilités de résoudre le problème et utilisaient leurs relations sociales de façon satisfaisante. Cette compétence faisant défaut, ils cherchent de l'aide auprès d'acteurs spécialisés et d'organismes désignés socialement à cet effet (Pray, 1947 ; Hahn, 1983 : 187).

L'action professionnelle des travailleurs sociaux a toujours été perçue comme une interposition - une interface - entre la personne-demandeure et I'établissement, qui est une émanation de la société. Placé entre le client et I'établissement, le travailleur social exercera, selon les situations et les problèmes, des rôles de conseiller, de facilitateur, d'intermédiaire, de médiateur et de protecteur, tantôt interprétant à l'intention des clients la position des décideurs-employeurs des institutions et établissements sociaux, tantôt expliquant l'univers des populations aux décideurs (Hernandez, 1985 : 32 ; McPheeters, 1971 ; Van Campenhout, 1989).

On peut dès lors appréhender la très grande complexité de l'action du travailleur social, au centre d'une pluralité d'univers, de perceptions, de valeurs, de contraintes... mettant en scène des acteurs et des institutions multiples (Bachman et Simonin, 1982 : 13-24). La situation d'interculturalité ne change pas cette complexité fondamentale de l'intervention sociale, elle l'amplifie. L'interculturalité ne fait que rendre les embrouillaminis plus fréquents, les risques d'incompréhension plus grands, les quiproquos plus nombreux, par l'effet des univers différents auxquels les acteurs appartiennent et qu'ils méconnaissent plus souvent qu'autrement réciproquement.

Le rôle du professionnel dans l'intervention interculturelle - à l'instar du proxène ${ }^{2}$ de la Grèce antique - prend tout son sens et toute son ampleur dans ce mouvement de va-et-vient entre la personne immigrante et les institutions de la société d'accueil. La qualité des échanges entre la personne immigrante et le professionnel implique que ce dernier travaille avec sécurité et souplesse. Une méthodologie d'intervention bien maîtrisée constituera la base de ces échanges, méthodologie qu'on définit comme un système articulé d'étapes nécessaires à la réalisation d'un projet ou d'une intention d'agir (Aubin, 1984; De Robertis, 1981 ; Du Ranquet, 1983), et qui comporte trois phases: la phase du début (la demande/la commande, I'exploration et I'analyse de la situation, la formulation de la problématique sociale), la phase du milieu (le plan d'intervention, I'action/le réajustement) et la phase de la fin (la terminaison et l'évaluation des résultats).

2. Personnage officiel de la cité chargé de s'occuper des intérêts des étrangers. 


\section{La phase du début}

\section{La demande / la commande}

Les différentes façons de soumettre un problème à l'attention de l'établissement et du travailleur social définissent le contexte d'intervention (Lebbe-Berrier, 1988 : 37, Prieur, 1989). Ainsi dans le cas où la personne se présente directement par elle-même et pour elle-même ou pour un proche ou, encore, lorsqu'une équipe de professionnels établissant un constat de problèmes sociaux fait une offre de service aux personnes en cause pour qu'ils cherchent ensemble des solutions, ces situations créent un contexte volontaire, c'est-à-dire une demande de la part du client. Par contre, si une personne s'adresse à l'établissement à la recommandation d'un professionnel ou si quelqu'un dénonce une situation qui doit être corrigée ou encore s'il y a des mesures de protection judiciaire ou de probation à l'égard d'une personne ou d'une famille, le contexte est non volontaire; on dira alors qu'il s'agit d'une commande.

Ainsi la perception de la réalité est « de nature non pas contemplative (observer la réalité comme de l'extérieur, objectivement), mais au contraire active, c'est-à-dire qu'elle est le fait d'acteurs socialement situés... caractérisés par une position et des dispositions». Selon sa position, chaque acteur en situation interculturelle perçoit la réalité sous un jour, sous un angle particulier, par des effets de perspective, de distance ou d'éloignement. Chaque acteur sera en position d'observateur et d'observé, et chacun aura tendance à se raccrocher à sa propre expérience pour analyser le comportement de l'autre, lorsque le comportement en question lui apparaîtra lointain ou peu intelligible. Chacun se prendra lui-même comme pôle de comparaison (Boudon, 1986 :105-118 et 146-149 ; Gunten, 189 : 26).

Quant aux dispositions, ce sont les ressources cognitives, affectives et éthiques que les acteurs possèdent et qu'ils utilisent pour interpréter la réalité. La conception du modèle de personne auquel l'intervenant adhère agit comme un filtre tout au long des rencontres professionnelles en situation d'interculturalité. Dans le modèle individualiste (prévalent en Amérique du Nord, en Europe...), la personne est caractérisée par l'émergence d'un moi comme une unité différenciée des autres et existant par elle-même, douée d'autonomie et d'indépendance; la personne à l'âge adulte doit procéder à la séparation physique et morale d'avec sa famille d'origine pour créer une autre cellule familiale ou du moins vivre indépendante des liens parentaux. À l'opposé, le modèle communautaire ou holistique (prévalent en Afrique, en Asie...) conçoit la personne en fonction de relations sociales ; dans ce modèle où il n'y a jamais de coupure du milieu familial d'origine et du groupe d'appartenance, c'est le sentiment du nous qui domine et non celui du moi. Les liens hiérarchiques reliant les personnes à la famille, au groupe ainsi que leur 
participation à la communauté et leur fidélité au groupe y sont valorisés. La solidarité est un impératif auquel personne ne peut se soustraire : on y fait appel pour la maladie, le deuil, I'éducation des enfants, etc. (Cohen-Emerique, 1990; Dumont, 1978).

À la réception du dossier, avant de rencontrer le client, le travailleur social peut amorcer une réflexion en profondeur sur la rencontre interculturelle. Il se pose des questions. Qu'est-ce que je connais objectivement de cette culture (situation géographique, histoire, langue...)? Ignorance totale? "Prêt à penser»? Folklore? Stéréotypes culturels? Au bureau, y a-t-il une personne-ressource que je peux consulter? Qu'est-ce que je ressens? Insécurité, anxiété, peur? Peutêtre fascination, hâte, intérêt... Identifier ce que me fait vivre le fait d'avoir ce dossier. Cela donnerait le temps d'en prendre conscience afin que ces schèmes n'introduisent pas un biais dans l'intervention.

Pour tous les scénarios du démarrage de la rencontre interculturelle, I'intervenant verra à soigner l'aménagement du local, sa présentation personnelle et professionnelle, la réception des tiers qui accompagnent le migrant.

L'aspect formel de nos bureaux peut être froid. Nos bureaux marquent souvent la distance face à l'autre, et ils peuvent rendre difficile l'amorce de la relation. Les orientations que la tradition professionnelle tenait pour importantes: local agréable, accessible rapidement à l'entrée, égayé et comportant une touche personnalisée sont plus que jamais valables pour une clientèle dont la socialisation est marquée par l'importance des liens personnels. La question de l'habillement peut également prendre de l'importance. Nous pouvons être " agressants " si notre habillement est trop étrange, tout particulièrement pour certaines cultures.

Il est important au moment des premiers contacts avec des clients de diverses ethnies d'appeler les personnes par leur nom au complet: dire Monsieur, Madame, les vouvoyer et employer les formules d'usage, par exemple en donnant une poignée de main ou en faisant d'autres gestes appropriés de politesse (Zastrow et Kirst-Ashman, 1987 : 513).

Préciser clairement par des phrases courtes notre fonction. Présenter l'établissement comme ce qu'il est, une institution aidante, et sa compétence dans les limites de sa mission. Le client arrive comment? Seul? En groupe? S'il vient en groupe, qui paraît être la personne significative? Si les personnes sont en contexte volontaire, la façon de voir l'intervenant va être différente. Si le contexte est humiliant ou non volontaire, elles se sont apporté du support. Est-ce un soutien naturel? Pourrions-nous nous en faire un allié? Qu'est-ce que nous ressentons? La structure sociale veut que les parents biologiques soient impliqués, mais, pour le client, quelqu'un d'autre peut avoir l'autorité morale. Il nous faut repenser d'autres façons de faire: des salles plus grandes ou encore la capacité pour le travailleur 
social de fonctionner en promiscuité. Nous sommes habitués à un champ spatial - " un territoire... ». Apprendre aussi à fonctionner avec d'autres interlocuteurs que juste la famille nucléaire.

\section{L'exploration et l'analyse de la situation}

En intervention culturelle, il est primordial de prendre le temps d'explorer et $d^{\prime}$ analyser la situation du migrant - ses conditions objectives de vie, ses logiques endogènes, ses réseaux sociaux d'appartenance et de rattachement -, et de mettre en place un espace intermédiaire, un espace de travail permettant des échanges avant d'arrêter un plan d'action.

Les conditions objectives de vie, ce sont des éléments matériels, concrets, objectifs, en somme des ensembles de faits qui caractérisent un système: individu, famille, groupe, communauté, etc. Elles ne relèvent pas de la volonté $d^{\prime}$ 'une personne et sont pour ainsi dire des contraintes de situations. Elles englobent les caractéristiques personnelles (sexe, âge, orientation sexuelle, statuts et rôles, état matrimonial, nombre d'enfants à charge, etc.), les caractéristiques développementales (caractères morphologiques: poids, taille; santé physique: maladie, handicap... et santé mentale; niveau de scolarité, qualification professionnelle et langues parlées; histoire de vie, antécédents familiaux et projet prémigratoire: motifs et conditions de départ du pays d'origine, acceptation ou non par l'entourage, attentes et projets de vie dans la société d'accueil), les conditions sociales (origine ethnique, nationalité, religion, statut migratoire (le migrant reçu, parrainé, réfugié, autre...) et, enfin, les conditions économiques (le cadre de vie: logement, nombre de pièces, qualité, coût ainsi que quartier et type de voisinage; l'emploi : activités, horaires, qualité de vie au travail, transport ; le revenu: salaire, allocations sociales et avantages sociaux divers, endettement) (Khon Feuermann, 1978 : 153 ; Bertot et Jacob, $1991: 43-45$ ).

Les logiques endogènes (du grec endo, en dedans) correspondent aux façons de penser, de sentir et d'agir d'une personne (ou d'un groupe ou d'une population). On peut les voir comme le vécu subjectif $d^{\prime} u n e$ personne (d'un groupe ou d'une communauté), c'est-à-dire l'ensemble de ses perceptions, sentiments, attitudes et comportements (Ampleman et al,. 1983; Moreau, 1991 : St-Arnaud, 1983), ou comme un cadre de référence, telle la grille de lecture qu'une personne s'est forgée à partir de son enfance et qu'elle a modifiée au fil de ses appartenances à d'autres groupes de référence (Bilodeau, 1982 ; Fisher et Ury, 1981). Elles peuvent aussi être associées à la rationalité de l'acteur (Assogba, 1991), comme la capacité à prendre des décisions compatibles avec les éléments d'une situation dans un contexte social donné (Ampleman et al,. 1983; Bilodeau, 1982 : 230 ; Fisher et Ury, 1981 : 46-50 ; Moreau, 1991 :113 ; St-Arnaud, 1983 : 1719). L'observation des vêtements, des rites de communication et d'hospi- 
talité, de l'organisation de l'espace familial ainsi que l'écoute attentive du discours de la personne migrante - mots clés, expressions particulières, citations de proverbes, histoires édifiantes ou allégories - constituent une source importante de renseignements sur les logiques endogènes de cette personne (Camilleri et Cohen-Emerique, 1989 : 96 ; Cohen-Emerique, 1980 : 134).

À ce sujet, l'intervenant en contexte interculturel doit prendre en considération le fait que les comportements adoptés par les clients nordaméricains pour parler de leurs problèmes psychosociaux de façon directe et souvent sur la place publique sont, pour de nombreux ressortissants de pays d'Europe, d'Afrique et d'Asie, choquants, brutaux, offensants pour la personne elle-même, pour sa dignité et même pour la dignité de celui à qui elle se confie. Le salaire, la sexualité, les problèmes conjugaux et familiaux sont des sujets tabous qu'on n'aborde qu'avec une grande réserve, dans la plus stricte intimité. La parole elliptique ou la circonlocution sera utilisée, ou encore les proverbes, les fables, les références à la Bible, au Coran, aux Livres sacrés. «Cette parole apparaît quand il faut dire quelque chose sans dévoiler complètement le secret de ce que l'on dit, ou aborder certains sujets délicats sans blesser personne» (Barel, $1982: 24)$.

Par ailleurs, il faut éviter de centrer son attention sur les motivations, les traits de personnalité, le passé, etc., au détriment de l'environnement physique et social, c'est-à-dire le cadre de vie du migrant, son insertion dans une multiplicité de sous-groupes, les dimensions socioculturelles (Murry, 1976 ; Laurent, 1988). L'analyse des réseaux sociaux auxquels I'immigrant se rattache devra d'abord porter sur les réseaux primaires ou de proximité, qui touchent l'ensemble des relations associées à la famille (la famille nucléaire, la parenté, le clan), au voisinage (le quartier, la rue, la cour commune, I'immeuble, l'étage, etc.), à l'amitié (l'ethnie, le village, la région, l'église) et à la camaraderie (la classe, le bureau, l'usine, le centre sportif, etc.).

On a du mal en Occident à percevoir plus particulièrement le rôle de la famille élargie dans la vie des migrants issus de sociétés traditionnelles où les beaux-parents, le frère aîné, les oncles et les tantes peuvent intervenir dans la vie du couple et en matière d'éducation des enfants... Ces personnes peuvent accompagner le client au bureau ou être présentes au moment des visites à domicile. Généralement, ce sont les aspects contraignants de la famille élargie que l'on note (entrave à la liberté individuelle, pénétration dans la vie privée du couple, nuisance à l'identité des personnes...) et non les aspects positifs (source de sécurité, solidarité, socialisation des enfants, protection contre les étrangers, etc.) (Cohen-Emerique, 1986). La famille élargie est, comme tout système, à la fois source de tensions, d'aliénation et source de possibilités, de loyauté. Les processus d'échanges avec le réseau primaire peuvent se révéler inadéquats du fait d'un blocage dans la 
communication ou d'attentes inconsistantes de part et d'autre ou encore du fait de relations d'exploitation. Ce n'est que lorsqu'il parvient à connaître, relativement au problème du client, ce que sont ces influences et comment elles agissent sur le client que le travailleur social peut déterminer dans quelle mesure et dans quelles conditions on peut agir sur elles, comment on peut annuler leurs effets néfastes ou accroître leurs effets bénéfiques (Germain et Gitterman, 1980 : 8 ; Leonard, 1976; Perlman, 1973 : 29).

Les réseaux secondaires renvoient pour leur part aux relations qu'une personne établit par sa participation à diverses institutions et organisations de la société. Ces relations existent sur des bases fonctionnelles; elles sont formelles lorsqu'elles sont liées à des institutions publiques ou semi-publiques et informelles si elles sont rattachées à des associations ou à des groupes communautaires.

En somme, pour l'ensemble des réseaux primaires et secondaires, il s'agit de vérifier la manière dont les liens sociaux sont mobilisés ou actualisés en cas de problème et la façon dont ils agissent dans la vie des migrants.

Tout au long de l'exploration et de l'analyse de la situation, I'intervenant doit prévoir un espace intermédiaire ou un espace de travail, c'est-à-dire un dispositif de paroles et d'échanges entre lui et le client où il sera possible de décoder les demandes pour s'interroger sur les façons de faire de chacun (Lebbe-Bernier, 1988: 36). Il s'agit en somme de l'empathie qui, en situation interculturelle, est basée autant sur la différence que sur la similitude et qui amène intervenant et migrant à se situer en tant que personne par rapport à une autre personne (Brunel, 1989 : 9-94. Cela suppose que le travailleur social ait amorcé un double processus de décentration de ses propres modèles (personnels et professionnels) et de relativisation de ses valeurs. valeurs notamment liées à l'autonomie et à l'indépendance de la personne, à la primauté de la famille nucléaire, à la conception égalitaire homme-femme, à l'anti-autoritarisme des relations parents-enfants, etc. Valeurs qui caractérisent, de façon générale, les pays occidentaux et dont I'intervenant est porteur (Das, 1988 : 31-55; Prieur, 1989; CohenEmerique, 1990 : 27).

C'est dans la mesure où les fondements de sa sécurité, de son équilibre et de son identité seront respectés que l'immigrant pourra aborder et intégrer les éléments de la nouvelle culture de la société d'accueil et d'une certaine manière y adhérer. Le travailleur social, en tant que représentant de cette culture, sera une personne significative dans ce processus de transformation. De plus, le travailleur social qui manifeste, par son attitude, du respect à l'égard de l'appartenance socioculturelle du migrant favorisera, par analogie, un processus de perméabilisation des frontières au nouvel environnement, ce qui favorisera une diminution du sentiment de menace et d'isolement chez le migrant (Barudy, 1989 : 24-26; Cohen-Emerique, 1980 : 138-139). 
Par ailleurs, la capacité de l'intervenant à traduire la réalité de la société d'accueil au nouvel arrivant est d'une importance capitale pour le processus d'intégration de ce dernier. Créer des liens de chaleur, de compréhension, de solidarité avec un représentant du pays d'accueil est essentiel pour le migrant nouveau, et cela peut constituer une expérience qui s'inscrit dans des liens plus profonds et intimes que de simples rapports professionnels. Cette expérience humaine représente pour tout migrant un atout important comme premier jalon dans la construction de son nouveau territoire (Compher, 1989 : XVIII). Cela n'est pas donné au départ et suppose un réel engagement de la part de l'intervenant.

En contexte volontaire (Caillé, 1984 : 355 ; Lebbe-Berrier,1988 : 37), créer un espace intermédiaire consiste, entre autres:

- à s'enquérir des événements qui ont pu déclencher la démarche d'une demande: vérifier ce que le client a pu faire auparavant pour résoudre la difficulté, avec l'aide ou non de parents, d'amis, de divers professionnels et d'organisations.

La première demande va donc souvent être concrète, mesurable par rapport à un service. C'est probablement à défaut d'un réseau propre que le service social va être sollicité. C'est la brisure avec le réseau naturel d'aide, l'absence de solidarité primaire: famille élargie, voisinage... Quant aux problèmes psychosociaux, ils vont surgir plus tard. Identifier quelles sont les tentatives de solution déjà faites. Lorsque les migrants se présentent eux-mêmes, c'est souvent pour un besoin concret de survie, parce qu'ils ont tout essayé et qu'ils viennent en extrême limite : une référence de l'école, une maladie. Très souvent, c'est par la connaissance d'une personne en particulier, ...l'importance aussi du bouche à oreille.

- à replacer la demande dans une perspective de compétence du client. Au lieu de disséquer les données motivant la demande, on revalorise la perspective de la demande grâce à l'acceptation inconditionnelle de ses prémices... Faire une demande est toujours une expérience douloureuse, si bien que la revalorisation des prémices de la demande et la prise en considération de la douleur inhérente à celle-ci sont un moyen d'investigation du système demandeur

Reconnaître la compétence de l'autre à dire son problème. Accepter de ne pas tout comprendre. Reconnaître l'importance de «traduire » les lois et exigences de la société d'accueil pour tous les citoyens. Respect en tout temps du vécu et de l'expérience de l'autre. Honnêteté et intégrité dès le début. Ne pas doser la qualité de sa relation, tout offrir en tenant compte de nos limites.

- à créer une atmosphère de respect vis-à-vis du problème symptôme, qui peut jouer un rôle de protection de quelqu'un ou de quelque chose - 
rôle de protection qui se révèle, pour toutes sortes de raisons, inefficace ou gênant - et ne plus être toléré par la famille ou un membre extérieur, et qui l'amène à solliciter l'aide d'un professionnel ou d'un organisme; et enfin définir un contexte d'intervention commun à toute la famille ou à tous les acteurs en présence.

La démarche la plus naturelle semble, dans un premier contact, $d^{\prime}$ accepter ce qui s'offre à nous. En effet, dans un premier temps, le client est là, c'est lui qui dit ce qu'il veut. Exprimer si l'on ne comprend pas, dire son ignorance de la culture et inviter le client à nous faire connaître ce qu'il sait. Cela le renforce dans son identité d'origine, ce qui le rend moins vulnérable. Le choc culturel pour l'intervenant devient ainsi une brèche par laquelle il peut accéder à la compréhension de l'autre. À travers cela, il faut créer un lien et le maintenir. C'est ainsi que l'on transmet le message que l'on est prêt à mettre de l'énergie pour eux. Un indice pour nous assurer de la pertinence de ce que nous apportons, c'est la participation du client au processus.

En contexte non volontaire (Lebbe-Berrier, 1988: 52; Pauzé et Petitclerc, 1986; Selvini-Palazzoli, 1984 : 89-99; Tilmans Ostyn, 1985 : 344), créer l'espace intermédiaire implique:

- de reconstituer avec le client le circuit des pressions et des demandes de la part des autres, circuit qui a provoqué leur rencontre, et de consacrer un temps où le client pourra exprimer ce qu'il comprend de la demande des autres, ce qu'il croit que les différentes personnes qui lui ont conseillé cette démarche espèrent de lui et de cette démarche, ce qu'en pensent éventuellement les autres membres de la famille; ce qu'il pense que le travailleur social va faire, à la suite de cette demande; puis, de vérifier avec le client si, pour lui, il y a problème et, si oui, quel est ce problème

...déceler si le client ressent un malaise dans la situation qui semble problématique et s'il y a quelque espoir que sa situation puisse changer. Sans ces deux éléments, il n'y aura pas de motivation et donc d'amorce de changement. Une des difficultés importantes, c'est lorsque notre perception du problème ne coïncide pas avec celle du client. La difficulté est encore plus grande lorsqu'il s'agit d'une convocation que le client a reçue et qu'il vient à reculons. Il se pourrait que pour lui les difficultés se traitent à l'intérieur de la famille et que l'on n'ouvre pas à l'extérieur. Souvent ils ignorent l'existence d'un service social gratuit.

- de recadrer à l'intention du client la démarche / demande « du client envoyé par un référent comme preuve de compétence, de force et d'investissement» (Tilmans Ostyn, 1985). Si nous ne procédons pas à ce recadrage positif de la démarche et si nous ne nous interrogeons pas 
sur le sens de l'envoi au service social et comment il pourrait être interprété par les proches, nous nous plaçons comme exécutant du référent, limitant notre champ d'action et celui des membres de la famille.

Cependant, dans tous les cas il est important d'amorcer l'analyse de la situation en donnant au client : le pouvoir de "se dire»: qu'estce qui le dérange? Lui laisser la possibilité de formuler sa propre demande et s'assurer qu'on l'a bien compris. S'il ne réussit pas à la développer, demander des clarifications. S'il met des limites, voir avec lui pourquoi . Dans certaines catégories, comme pour la clientèle Service aux migrants et immigrants avec aide financière, cela peut être difficile, car ils ne savent pas ce qu'ils devraient dire ou ne pas dire pour obtenir l'aide financière. Dans tous les cas, lui faire saisir que l'on partage sa souffrance et que son inconfort est compris. Montrer notre intérêt à l'égard de sa situation.

En contexte de judiciarisation (Lebbe-Berrier, 1988 : 38; Poisson, 1988 : 324325 ; Evequoz, 1984 : 94), créer l'espace intermédiaire signifie:

- expliciter clairement les règles du jeu contraignantes tant pour la famille qui n'a pas le choix de refuser cette intervention que pour l'intervenant qui a l'obligation de faire rapport au juge des enfants.

Il faut situer sans équivoque ce contexte d'obligation. Cependant, pour donner du sens à cette intervention, le travailleur social devra aider le migrant à trouver des points de repère et des correspondances, dans sa culture d'origine, "à ce non-négociable». À travers cette intervention, une perception plus adéquate de la société d'accueil est rendue possible.

- de reconnaître leur non-demande : I'attitude de déni ou de rejet.

Il peut arriver qu'à l'évaluation, I'interprétation des situations puisse être différente. Là où nous voyons un problème, le client, lui, peut ne pas en voir. II nous faudra alors discerner le point sur lequel nous ne pouvons faire de concession : ce qui serait inacceptable pour l'ensemble de la population, de la société du pays d'accueil. Prendre du recul avant de pouvoir faire "digérer» ce qui de prime abord paraît inacceptable au client. Plus ces valeurs sont ancrées dans une vision que l'autre a du monde, moins le client permet que nous les mettions en questions. Souvent même elles sont plus inconscientes et peuvent heurter nos propres valeurs plus ou moins conscientes. Dans notre cueillette d'information, nous risquons ainsi d'embrigader l'autre dans des catégories "prêt-à-penser».

- de stimuler l'émergence d'une demande. L'intervenant peut d'entrée de jeu déclarer: je suis mandaté pour intervenir; nous allons tenter ensemble de comprendre ce qui fait que vous êtes là. Le client doit se sentir respecté dans sa cohérence: il s'agit pour l'intervenant d'apprécier positivement, 
dès le début, l'effort fait par le client pour résoudre les problèmes, en donnant à celui-ci l'espace nécessaire pour se sentir accepté et valorisé dans la relation avec l'intervenant et donc ouvert au dialogue.

Nous sentons-nous en situation de domination ou, au contraire, menacés dans le "contrôle" de notre entrevue, surtout si les gens parlent leur langue? Le fait de cerner ce qui se passe en nous permet de prendre de la distance par rapport à cette autorité et d'accueillir le fonctionnement de la culture particulière en présence de laquelle nous nous trouvons. Dans un deuxième contact, nous aurons tout loisir de défricher, d'évacuer, de ne pas nécessairement donner crédit à tout ce qui est dit... Alors, nous pourrons expliquer, apprivoiser, apaiser, sécuriser dans les limites de nos mandats. Si le client se sent accueilli, si le contact est bon, nos exigences pourront être plus possibles. La première impression que les clients ont du Service, ils vont la garder. Souvent c'est pour la première fois qu'ils ont affaire à notre service. Il est important que nous leur interprétions la loi faite pour tous. Garder pourtant une ouverture spécifique, ne pas en mettre trop. Être vigilant pour le langage, le vocabulaire. Éviter un jargon qui souvent reste obscur entre deux services pour des Québécois. Vérifier s'ils ont bien compris. S'agit-il des mêmes réalités? Développer l'expertise du sens du langage.

\section{La problématique sociale (le diagnostic social)}

En interculturalité, la position du problème se situe nécessairement dans un contexte de transition que représente le phénomène migratoire, qu'il soit volontaire ou non volontaire, transition qui implique une translocation et une transculturation (Casse, 1978 ; Sayad, 1989; Serrano, 1989 : 313 ; Vasquez, 1984 : 119). La translocation, c'est le fait d'être propulsé en peu de temps de son environnement traditionnel à un autre environnement, alors que la transculturation est le fait de la rencontre de deux cultures, deux modes de pensée, deux modèles de référence, et le fait des modifications indirectes du comportement chez les immigrants par la société d'accueil, dans une gamme de possibilités d'adaptation plus ou moins vastes et complexes.

La transculturation implique un double processus de déculturation et de néoculturation. La déculturation (une émigration de...) comporte la perte ou l'érosion de la culture d'origine. L'émigré aura, entre autres, à vivre la désinsertion personnelle et familiale de sa société d'origine et à faire l'expérience émotionnelle de la séparation et du déracinement, sans parler de la perte de ses cadres sociaux familiers, de ses anciens objets d'amour et de la perte de la reconnaissance accumulée dans son pays d'origine. La néoculturation (une immigration ici...) est l'acquisition d'une culture nouvelle et différente. L'immigrant (et sa famille) devra faire face à la transplantation dans 
un espace étranger, à l'insertion dans un cadre symbolique nouveau, à l'acquisition de nouvelles compétences, à l'expérience quotidienne de vivre la suspicion que suscite sa présence dans le pays d'accueil.

La transculturation provoque souvent des perturbations aux plans personnel et familial, entre autres (Barudy, $1989: 22$; Bertot et Jacob, 1991 : 50-53; Serrano, $1989: 315-316)$ :

- la diminution du statut socio-économique pour plusieurs migrants dans le pays d'accueil par rapport à celui existant antérieurement: difficulté à trouver un emploi selon ses compétences réelles, disqualification des diplômes, acceptation d'emplois «peu valorisés ou à bas salaire, etc. » ;

- la perte d'estime de soi et une baisse du statut social, surtout pour les hommes à qui est attribué le rôle traditionnel de pourvoyeur économique de la famille, où le modèle patriarcal est très valorisé;

- I'infantilisation et une position de dépendance des adultes par rapport aux institutions du pays d'accueil. La reconnaissance des rôles sociaux et professionnels que les adultes avaient dans leur pays d'origine a disparu. Dans le pays d'accueil, les migrants essaient d'être définis et respectés comme des adultes, mais paradoxalement, dans le nouvel environnement ils ne connaissent souvent ni la langue du pays, ni les coutumes, ni même le minimum pour comprendre le fonctionnement du pays; ils sont renvoyés à un état d'infantilisation et se trouvent dans une position de dépendance et d'assistance par rapport aux habitants du pays d'accueil et aux fonctionnaires de ses institutions;

- I'élimination, ou du moins la réduction de la famille étendue. Ce tissu de relations est une source importante de satisfactions affectives, de solidarité, d'aide et de soutien devant les difficultés de l'existence. La famille migrante devient donc plus vulnérable aux modifications de l'environnement;

- la dévalorisation de l'image parentale. L'adolescent de la famille migrante est amené à comparer le style de vie de sa famille, les mœurs propres à sa culture avec ceux du pays d'accueil. À travers le discours de la société d'accueil, notamment à l'école, l'adolescent se découvre étranger, appartenant à un groupe souvent dévalorisé, à tout le moins chargé de stéréotypes défavorables. L'adolescent recherche donc des modèles d'identification en dehors de sa famille ou de son groupe culturel d'appartenance. Il adopte souvent une attitude critique et défensive qui se traduit par une dévalorisation de l'image des parents qui représentent la culture d'origine.

- la parentalisation d'un des enfants. Il arrive souvent dans la famille qu'un adolescent - et même un enfant - soit le seul qui parle (lit et écrit) la langue du pays d'accueil. Celui-ci sert ainsi d'intermédiaire entre la famille et les 
diverses institutions sociales, école, mairie, voisinage, etc. Cette situation qui représente un pouvoir de parent ne correspond pas à son âge et à son statut.

- le renforcement des attitudes autoritaires. La perte du prestige social vécue par les parents est une source de souffrances; elle entraîne souvent une démission du rôle du père et le renforcement des attitudes autoritaires de ce dernier, dans la famille et à l'extérieur, comme moyen de compenser cette perte et comme un effort pour être reconnu en tant qu'adulte et partenaire.

L'immigration entraîne des mécanismes d'adaptation multiples de la part de l'immigrant (Barudy, 1989 :24-26; Cohen-Emerique, 1986 : 72 ; Sterlin, 1987 : 103). Ce sont:

- I'adaptation instrumentale, qui consiste à s'orienter dans l'espace géographique, à se vêtir correctement en fonction des saisons, à s'initier aux procédures administratives, à utiliser les moyens de transport, etc.;

- I'adaptation comportementale, où il s'agit de maintenir une distance spatiale et psychologique adéquate dans les rapports sociaux, d'éviter de gesticuler ou de rire de façon bruyante, etc.;

- I'adaptation culturelle, qui est liée aux normes et valeurs dominantes de la société d'accueil, soit au statut et au rôle de la femme, à l'autonomie de la personne, à l'émancipation, à l'égalité des droits, à la planification de la famille, à la liberté de la contraception, etc.

Un processus d'équilibration-insertion s'installera selon chaque migrant (Barudy, 1989 : 24-27 ; Campher, 1989 : 63-73 ; Malewska-Peyre, 1989: 125-129; Serrano $1986: 73-85)$ :

- soit par la stricte observation des normes de la culture d'origine: on assiste alors au maintien et au renforcement des fonctionnements préexistants. Au début, ces comportements peuvent être nécessaires pour faire face aux menaces de désintégration; leur rigidification, par contre, bloque les possibilités d'échange avec l'environnement nouveau et peut entraîner la formation de ghettos;

- soit par l'ouverture exagérée à la société d'accueil, c'est-à-dire l'assimilation: dans ce cas une grande perméabilité des frontières familiales peut provoquer chez les enfants et les adolescents un sentiment de non-appartenance;

- soit par le syncrétisme, qui consiste dans l'adoption de comportements extérieurs par intérêt ou par souci de paraître en accord avec la société d'accueil. On a l'exemple du père de religion islamique qui autorise sa fille à faire des études, mais qui la marie sans son accord; 
- soit par la synthèse ou l'adaptation fonctionnelle ou l'intégration critique, c'est-à-dire un dosage permanence-changement. D'une part, une équilibration permet le renforcement des structures et du fonctionnement familial d'origine, de façon à conserver et à utiliser le bagage expérientiel et culturel de la famille pour faire face à la crise et, d'autre part, une perméabilité des frontières familiales et individuelles sert à utiliser les énergies, informations et apports de la société $d^{\prime}$ accueil.

\section{Le plan d'intervention et l'action}

Les réajustements des comportements professionnels à cette étape dans I'intervention interculturelle porteront autant sur des attitudes qui traduisent le respect des différences entre immigrant et intervenant que sur des orientations d'actions qui tiennent compte des assises culturelles de l'immigrant. $C^{\prime}$ est tout spécialement vrai dans l'assistance que le professionnel apporte dans les conflits parents-enfants, de même que dans les situations de protection de la jeunesse et de délinquance juvénile.

Le respect de l'immigrant se traduira par la reconnaissance active de sa compétence. On a depuis longtemps en travail social accordé une attention toute spéciale aux relations professionnelles entre le travailleur social et le client, qui sont empreintes de rapports de domination et tenté d'y apporter les correctifs nécessaires (Bilodeau, 1980 ; Moreau, 1979). Ce qu'il s'agit de combattre en interculturalité, c'est I'hégémonie culturelle, ce sentiment de supériorité et d'arrogance occidentales (Bruckner, 1992) que des intervenants peuvent développer - souvent plus ou moins consciemment - face à des populations dites du tiers-monde. L'intervenant accepte alors de ne plus être le détenteur unique d'un savoir et d'un faire; il se présente aussi comme celui qui ne sait pas et qui cherche à découvrir auprès du migrant lui-même ou des représentants de sa culture les réponses à ses interrogations. L'intervenant recherchera donc de façon constante à proposer des solutions qui entrent en résonance avec les éléments de cette stucture unique qu'est l'immigrant, des solutions crédibles et praticables dans et selon le cadre de référence de ce dernier. (Bilodeau, 1980 : 438-458; Bruckner, 1992; Brunel, 1989 : 86 ; Carino, 1982 ; Moreau, 1979; Cohen-Emerique, 1980 : 137).

Laisser questionner nos façons de faire; plutôt que le savoir, viser à développer la confiance personnelle. II n'y a pas de recettes. II faut accepter de faire. L'identité professionnelle change et nous-mêmes nous nous transformons par notre pratique. L'identité personnelle, la sécurité personnelle permettent la négociation. Partir de l'autre personne, accepter de faire un bout de chemin. Être perméable : nous n'avons rien à imposer, rien à défendre. Accepter la différence, ne pas avoir peur des choix. Perçu comme "expert de l'aide», comment je me sens si je n'ai pas de réponse à apporter? L'autre 
va-t-il accepter de prendre le pouvoir? Notre expertise est précisément dans l'habileté à engager la relation; cela peut décontenancer le client quand on lui remet le pouvoir. Se redire que le client et nous voulons la même chose, même si nos visions du monde sont différentes : I'adéquation du service rendu au besoin du client. Dans nos désarrois, accepter que l'autre ne soit pas comme nous. Garder le respect pour lui. Nous finissons par acquérir une capacité de nous faire déstabiliser. Il est alors important de s'utiliser. Prendre pour point de repère les points de ressemblance. En situation interculturelle, déstabilisés en tant que professionnels par notre clientèle, nous pouvons admettre notre "faillibilité » et garder quand même notre sécurité en basant notre action sur des principes humanistes d'égalité, de respect, de partage réel de pouvoir.

Le respect de l'immigrant passe également par l'acceptation d'un cheminement différent de celui du travailleur social. «Beaucoup de professionnels se perçoivent comme ayant fait une trajectoire libérée des contraintes sociales d'origine, ou tout au moins d'avoir été capables de les dépasser grâce à leur personnalité et à l'affirmation de soi »(Cohen-Emerique, 1990 : 20).

Dans la rencontre interculturelle, la question de valeurs et, souvent au Québec, la religion, interfèrent et constituent un point sensible qui nous entraîne dans des difficultés tant d'ordre affectif que "cognitif ». Souvent pour certains immigrants, ce qui est exprimé c'est un idéal religieux inculqué, mais ce que l'on voit, c'est une dichotomie: la personne se comporte sans tenir compte de l'idéal. Comment navigue-t-elle entre les deux? II semble que le "marchandage» soit courant. II nous faut être très vigilant pour prendre de la distance par rapport à notre propre ethnocentrisme (non seulement civilisation judéo-chrétienne, mais encore notre teinture locale) et voir comment la religion teinte l'éducation, la perception de la société et finalement le comportement de l'autre. La vulnérabilité des nouveaux arrivants lorsqu'ils vivent beaucoup de problèmes d'adaptation les rend des proies faciles à toutes sortes d'idéologies ou de mouvements intégristes. La religion peut devenir ainsi une béquille à un fonctionnement. Elle sert à adapter à un contexte social perçu comme menaçant. Au Québec, parce que la libération par rapport à une religion qui dicte un fonctionnement est récente, nous vivons l'inverse de façon très intolérante. Les changements de valeurs au Québec ont concerné le sens du sacré; du respect et de la vénération, on est passé au tu et à toi. Les nouveaux arrivants nous reprochent la désacralisation de notre société, un nivellement par le bas.

Par ailleurs, I'institution où le travailleur social intervient est porteuse de valeurs. La clientèle migrante sera toujours vue «à travers le prisme déformant de l'institution où exerce le travailleur social et, quoi qu'il fasse, ce dernier sera toujours perçu par le migrant comme le représentant d'un service donné 
"inséré" dans la société d'accueil, avec des pouvoirs plus ou moins importants d'aide et de contrôle social » (Cohen-Emerique, 1990). Les notions d'efficacité amènent souvent les intervenants à bousculer les étapes d'intervention, laissant peu de place au «temps pour surmonter les problèmes linguistiques, temps pour demander des éclaircissements, temps pour comprendre et se faire comprendre, temps pour transmettre aux autres partenaires s'occupant de migrants la manière de prendre en compte leurs identités, temps enfin pour réfléchir à sa propre approche » (Camilleri et Cohen-Emerique, 1989 : 112; Douyon, 1988).

Outre que nos propres valeurs ne soient pas toujours claires pour nous, il faut compter avec les "règles institutionnelles ». II nous faut prendre rapidement une orientation lorsqu'un dossier arrive en évaluation, dans des délais très précis, et travailler en concertation avec les autres partenaires institutionnels et la communauté. Certes, nous avons, pour le moment, à composer avec les règles institutionnelles, mais nous avons également l'obligation professionnelle d'en souligner les effets pervers, surtout l'inadéquation de ce fonctionnement en situation d'intervention interculturelle où la notion de temps est une dimension nécessaire au $b$ a ba de l'intervention.

Toutes les interventions en interculturalité devraient viser à recréer le tissu social de l'immigrant en sollicitant la participation active des réseaux primaires, les considérant comme des partenaires qui peuvent apporter éclairage, soutien, assistance. Ainsi I'intervenant valorisera la retribalisation, cette tendance des groupes nationaux à se concentrer dans certains espaces privilégiés ou à participer à des activités liées à leur histoire, ce qui assure en partie le maintien de l'identité culturelle du migrant; il aidera les migrants à s'insérer dans des groupes de pairs et dans les divers organismes non gouvernementaux pour obtenir du soutien psychologique, technique ou matériel; et enfin il élargira l'entraide au moyen de rencontres entre les habitants du pays d'accueil et les immigrants (Barudy, $1989: 28$; Bertot et Jacob, 1991 : 53 et 195 ; Cohen-Emerique, 1990: 28; Bastide, $1973: 29$; Douyon, 1988 : 14-20 ; Hémon, 1988 : 328-330.

En outre, dans les conflits parents-enfants, l'intervenant fera tout pour maintenir les liens familiaux (Cohen-Emerique, 1990). Il devra se départir de la croyance qui valorise l'autonomie, l'indépendance, la prise en charge personnelle comme signe de maturité d'une personne... Dans beaucoup de sociétés non occidentales, ce qui est important, c'est « la pluralité des éléments constitutifs de la personnalité et la fusion de l'individu dans son environnement ou son passé » (Bastide, 1973) et, pour nombre d'immigrants, être relié à la famille et aux amis, c'est non seulement souhaitable, mais aussi considéré comme une source majeure de soutien économique et social (PiglerChristensen, 1986: 11). L'intervenant devrait davantage aider les migrants à trouver leur équilibre dans la préservation des deux dimensions: individualiste 
et communautaire. Il évitera de poser les problèmes de façon dichotomique et de pousser les jeunes à des choix : société d'accueil ou société d'origine? Il soutiendra le processus de reconnaissance et de valorisation des multiples appartenances, même si elles sont sources de conflits, afin justement de les dépasser ou tout au moins de les assumer. II aidera les adolescents à accepter les dissonances, les contradictions et les tensions, inhérentes au processus de changements culturels (Chorbal, 193 ; Cohen-Emerique, 1990). En fonction des situations et des acteurs en présence dans ces conflits, l'intervenant fera appel aux capacités de créativité et d'invention; il aidera à développer des stratégies multiples d'intégration «groupe d'origine et société d'accueil » par la pratique du compromis ou des concessions ou même des omissions, des simulacres. L'intervenant pourra, par exemple, conseiller à un adolescent d'adopter un comportement à l'extérieur et un autre à la maison pour répondre aux vœux de ses parents; ou encore, dans le cas d'une adolescente qui cherche à s'émanciper, il envisagera d'aider cette dernière à aménager la réalité pour cacher des faits et gestes inacceptables dans sa famille d'origine et son groupe ethnique (Cohen-Emerique, 1990). Si cela s'avérait nécessaire, on pourrait même s'inspirer d'une pratique de simulacre où, à l'instigation d'intervenantes d'un organisme non gouvernemental de Guinée, on a mis au point, avec la complicité des mères, un semblant de clitoridectomie. Cris, pleurs, rituels, tout y est respecté, si ce n'est que l'aiguille utilisée est stérile... et courbée. Cette ruse permet à la jeune fille d'en sortir sans mutilation morale et physique (Lamontagne, 1989: 38).

Dans les situations de protection de la jeunesse, I'intervenant devrait prendre en considération que tout migrant se comporte selon les référents de son univers socioculturel d'origine. L'intervenant doit donc replacer les méthodes éducatives dans ces cas à la fois dans leurs contextes socioculturels d'origine et transculturels. Il est sage dans ces situations de consulter des professionnels de même origine culturelle que les migrants concernés pour essayer de saisir les codes familiaux. L'intervenant doit instaurer et maintenir un dialogue actif et un processus d'échanges concernant les écarts entre les deux cultures. Il doit se soucier des parents fragilisés par le processus d'acculturation, autant que par leur position de minorités dans la société d'accueil (Cohen-Emerique,1984 : 203-204 ; Compher, 1989; Hémon, 1988 ; Pigler-Christensen, 1988 ; Serrano,1989 : 315).

La «sous-culture» propre à la profession est souvent imposée aux clients en interculturalité: comme les critères de ce qui est appelé "négligence », très important en matière de protection. Non seulement il faudrait être attentif aux valeurs véhiculées par la profession qui sont souvent celles d'une certaine catégorie de classe dans la société, mais encore le praticien devrait être "connecté" à ses propres émotions, ses propres valeurs qui sont bousculées. C'est souvent le praticien qui est en état de choc culturel, même dans sa 
propre culture. Il est impératif qu'il en soit conscient. En France, par exemple, la notion de danger est très différente de la nôtre: ceux qui travaillent avec les gitans ne se posent pas de questions si sept personnes vivent dans un $31 / 2$. La situation est "pitoyable" pour qui ? Si la protection de l'enfance devait se mêler de cette situation, jusqu'où devrait-elle aller? Au Québec, nous n'acceptons pas la promiscuité, mais nous acceptons le chômage! Qu'est-ce qui est un danger pour la société? II nous faut redécouvrir des techniques plus collées au concret, des façons d'interroger et d'écouter qui vont conduire à des solutions nouvelles. On découvrira lors d'une visite en protection qu'il s'agit d'une bonne mère plus adéquate que les solutions de rechange que nous apporterons.

L'intervenant s'habituera à ne jamais essayer de négocier les valeurs ou les idéologies d'une personne: il vaut mieux se centrer sur les aspects tangibles ou les conséquences concrètes des positions idéologiques. II cherchera davantage à créer avec la personne immigrée toute une série de petits consensus, consensus qui tendent, plutôt de gré que de force, à l'intégrer dans un contexte où certaines normes sociales ne peuvent être ignorées (Pfeffer et Jones, 1976 ; Arnauld, 1989 : 128-129). Par exemple, la répudiation, la polygamie, l'excision, qui sont des règles du droit coutumier musulman, deviennent inapplicables en Europe et en Amérique du Nord (Actualités sociales hebdomadaires, 1992: 25). Dans ce cas, il est nécessaire d'éviter le dirigisme, en sollicitant plutôt la participation active de la personne par des remises en question et en cherchant des solutions ad hoc.

Si la notion du pouvoir est ambiguë et que lors de ses premiers contacts le client doit faire des démarches en démocratie, cela peut être destructurant pour lui et son comportement peut ne pas nous paraître acceptable. Comment notre réaction sera-t-elle reçue par l'autre? Où commence l'interculturel? Comment conjuguer la réaction de l'autre à l'histoire d'un pays dictateur? Qu'est-ce qui est culturel? Qu'est-ce qui est personnel? Devant un comportement inacceptable, il ne s'agira pas de perpétuer une attitude dictatoriale en réagissant de façon autoritaire. Une sécurité intérieure et une assurance peuvent permettre une confrontation, une correction de la situation en «expérience interculturelle». C'est ainsi que la rencontre du professionnel avec son client se situe en interculturalité, ce qui en souligne le caractère interactif, en préservant la différence de l'un et de l'autre. C'est une rencontre expérientielle. Par ailleurs, il arrive assez souvent que le travailleur social veut se protéger en signalant des situations dites de négligences. L'ensemble du groupe est contre cette situation. II existe des alternatives aux signalements. Nous savons où sont les besoins. Nous ne devrions pas faire des zèle au-delà de notre mandat. 
Dans les situations de délinquance juvénile, l'intervenant doit se rappeler que dans les sociétés où le modèle communautaire prédomine, la personne est reconnue par ses appartenances et sa place dans le groupe en fonction de ses rôles et statuts, qui codifient sa conduite. Si elle ne s'y conforme pas, ce n'est pas seulement elle-même qui est mise en cause, mais aussi son groupe. L'adolescent par son acte de délinquance ternit ainsi I'image de tout son groupe d'appartenance et compromet l'honneur de tous. Ainsi un adolescent qui, par ses échecs scolaires ou sa conduite, provoque l'intervention des autorités du pays d'accueil porte atteinte au projet migratoire des parents qui y ont investi leurs espoirs d'une vie meilleure, parents dont une des consolations, c'est que les enfants réalisent ce qu'ils n'ont pu réussir. Dans ces situations, pour beaucoup de parents, c'est donc l'effondrement, d'autant plus que les institutions du pays d'accueil représentent souvent à leurs yeux des menaces à leur identité (Camilleri et Cohen-Emerique, 1989 : 84-85 ; Cohen-Emerique, 1990 : 14; Malewska-Peyre, 1984).

Il faut définir le besoin des jeunes en fonction de leur adaptation. C'est quoi leur besoin de sécurité, d'accompagnement? C'est quoi leurs besoins pour évoluer au moins minimalement dans leur nouveau contexte? Quel est leur projet migratoire? Dans quel contexte sont-ils arrivés au Canada? L'habileté qu'il faut développer, c'est le discernement. Il y a certes risques de confondre la culture - qui a bon dos - et les traits de personnalité. Au niveau de la délinquance, il y a un piège: difficultés d'adaptation? troubles de comportement? ou réelle délinquance? Très souvent, la méconnaissance du système, les nuances de langage et la complexité de l'appareil judiciaire font que des parents de jeunes délinquants sont complètement dépassés. IIs voient leur jeune partir pour un centre d'accueil, vivent l'événement comme un enlèvement de leur enfant. Un petit vol par un enfant immigrant et toute la déception des parents se déverse. "Je n'ai plus le contrôle de mon fils... je ne vous comprends pas... ». Et des jugements très sévères sont portés sur la société d'accueil.

\section{La terminaison et l'évaluation des résultats}

Dans cette phase, le professionnel doit établir le bilan de l'action professionnelle, c'est-à-dire évaluer les effets des stratégies et moyens sur la vie et la situation du client, et préparer ce dernier à mettre fin aux rencontres avec le travailleur social.

La terminaison de la relation d'aide en interculturel semble comporter une difficulté très particulière pour les intervenants. S'il y a transfert, le préparer et accepter de rester quelque temps. 


\section{CONCLUSION}

Depuis quelques années, la profession de travail social a été remise en question par le phénomène du multiculturalisme, de l'interculturalité. Les professionnels se sont sentis déstabilisés, parfois incompétents devant les situations de ces personnes et groupes venus d'ailleurs.

Dans ce document, nous avons essayé de considérer les comportements professionnels du travailleur social à la fois dans l'universel et dans le singulier. L'intervention sociale en contexte d'interculturalité s'appréhende dans une vue globale, holistique, comme à deux niveaux simultanément: en toile de fond, la perception de toute la complexité dynamique d'une personne (ou un groupe de personnes) en situation sociale et, en relief, dans une plus grande acuité, la situation particulière d'une personne qui fait face à des exigences dont souvent le sens même lui échappe.

La stratégie de fond de l'intervenant social a pour base l'abandon par les deux parties (intervenant et immigrant) des schèmes culturels rigides qui les empêchent de s'aventurer dans ces territoires inconnus de l'immigration avec un esprit d'ouverture et de créativité. Cette stratégie prend ses racines dans la conviction profonde que l'immigrant et sa famille sauront trouver les voies pour survivre dans la société nouvelle (Brunel, 1989; Capra, 1983:372).

C'est dans cette densité de réalités multiples que le travailleur social, sollicité par le client ou mandaté par l'organisation sociale, devra en toute maîtrise de sa méthodologie offrir le soutien qui permettra à la personne (groupe ou communauté) de rehausser sa compétence sociale dans le nouveau tissu social qu'elle doit se constituer du fait de la situation de transition que l'immigration lui impose.

\section{Références bibliographiques}

Actualités sociales hebdomadaires. (1992). «Le conseil des sages fixe de nouvelles règles pour l'intégration », $\mathrm{n}^{\circ} 1772,14$ février.

AMPLEMAN, G. et al. (1983). "Pratiques de conscientisation», Nouvelle optique, Montréal, p. 111-113 et p. 126-127.

ARNAULD, G. (1989). «Le conseil : comment être ou comment faire», Connexions, $\mathrm{n}^{\circ} 1$.

ASSOGBA, Y. (1991). "L'organisation communautaire avec des communautés locales en Afrique », dans L. Doucet et L. Favreau, Théorie et pratiques en organisation communautaire. Québec: Presses de I'Université du Québec.

AuBIN, A. (1984). Passons à l'action. Programme de promotion de la femme. Montréal: Secrétariat d'État. 
BACHMANN, C. et J. SIMONIN (1982). Changer au quotidien. Une introduction au travail social. Tome 2. Paris: Études vivantes.

BARBIER, R. (1975). «Implication, animation et recherche-action dans les sciences humaines», Connexions, ${ }^{\circ} 13$.

BAREL, Y. (1982). La marginalité sociale. Paris: Presses universitaires de France.

BARUDY, J. (1989). «L'utilisation de l'approche systémique lors de thérapies avec des familles de réfugiés politiques», Thérapie familiale, 1.

BERTOT, J. et A. JACOB (1991). Intervenir avec les immigrants et les réfugiés. Montréal: Éditions du Méridien.

BILODEAU, G. (1980). «Pour une réelle relation d'alliance travailleur social - client », Service social, vol. 29, $\mathrm{n}^{\circ} 3$.

BILODEAU, G. (1982). «Un outil nécessaire: la négociation », Service social, vol. 31, $\mathrm{n}^{\text {os }} 2-3$.

BOUDON, R. (1986). L'idéologie ou l'origine des idées reçues. Paris: Fayard.

BRUCKNER, P. (1982). «Le grand comparateur. Un entretien avec Louis Dumont», Le Nouvel Observateur, $\mathrm{n}^{\circ} 1417,2$ au 8 janvier.

BRUNEL, M.L. (1989). "L'empathie en counseling interculturel », Santé mentale au Québec, vol. XIV, nº 1.

CAILLÉ, P. (1984). «Quelques considérations sur la signification de la demande en thérapie familiale», Thérapie familiale, 4.

CAMILleri, C. et M. COHEN-EMERIQue (1989). Chocs de culture: chocs et enjeux pratiques de l'interculturel. Paris: L'Harmattan.

CAPRA, F. (1983). Le temps du changement, Monaco : Éditions du Rocher.

CARINO, L. (1982). "L'analyse des besoins», Service social dans le monde, 2.

CASSE, P. (1978). "Éco-éthologie de la coopération», Service social dans le monde, 2.

ChORBAL, M. (1983). «Espace communautaire. Aspect spécifique de l'activité physique du Maghrében », L'Évolution psychiatrique.

COHEN-EMERIQUE, M. (1980). «Éléments de base pour une formation à l'approche de migrants et plus généralement à l'approche interculturelle», Annales de Vaucresson, 17.

COHEN-EMERIQUe, M. (1986). Représentations et attitudes de certains agents de socialisation concernant l'identité des migrants et de leurs enfants. Colloque de l'ARIC, Sèvres (document miméographié).

COHEN-EMERIQUE, M. (1986). «Adapter la formation en travail social», Entretien de B. Sacks avec Mme Cohen-Emerique. Information sociales, $\mathrm{n}^{\circ} 1$.

COHEN-EMERIQUE, M. (1990). « Le modèle individualiste du sujet. Écran à la compréhension de personnes issues de sociétés non occidentales », Cahiers de sociologie économique et culturelle, 13.

COMPHER, J.V. (1989). Family Centered Practice. New York: Human Services Press.

CORPORATION PROFESSIONNELLE DES TRAVAILLEURS SOCIAUX DU QUÉBEC (1991). Les normes de pratique professionnelle des travailleurs sociaux. Montréal.

DAS, K. (1988). «Travail social et pluralisme culturel au Québec: des enjeux inexplorés », Interculture, 7.

DE ROBERTIS, C. (1981). Méthodologie de l'intervention en service social. Paris : Le Centurion. 
DOUYON, E. (1988). «De l'expertise à l'intervention en milieu interculturel », Interculture, $\mathrm{n}^{\circ} 7$.

DUMONT, L. (1978). «La conception moderne de l'individu», Esprit, n 2.

DU RANQUET, M. (1983). Les approches en travail social. Montréal et Paris : Edisem et Le Centurion.

EVEQUOZ, G. (1984). Le contrôle scolaire et ses otages : vers une approche systémique des difficultés scolaires. Paris : Éditions ESF.

FISHER, R. et W. URY (1981). Comment réussir une négociation. Paris: Seuil.

Germain, C.B. et A. GitTerman (1980). The Life Model of Social Work Practice. New York: Columbia University Press.

GUNTERN, G. (1989). «Éco-anthropologie et thérapie systémique: une nouvelle image de l'homme», Thérapie familiale, 1.

HAHN, G. (1983). «Les raisons d'être du travail social », dans Quel travail social pour notre temps. Mutations des besoins. Mutations des pratiques. Paris : Éditions ESF.

HeRNANDEZ, S.H. et al. (1985). «Integrated Practice: An Advanced Generalist Curriculum to Prepare Social Problem Specialists », dans Journal of Social Work Education, $\mathrm{n}^{\circ} 3$.

KHON FEUERMANN, A. (1978). Relations humaines individuelles et communautaires. $18^{\mathrm{e}}$ conférence internationale de I'action sociale à Jérusalem-Israël 1978. Vienne: Conseil international de l'action sociale.

LAMONTAGNE, I. (1989). "Grandeurs et limites d'une révolution sexuelle », Santé société, 1 .

LAURENT, D. (1988). "La santé: la maladie sociale » Service social dans le monde, 2-3.

LEBBE-BARRIER, P. (1988). Pouvoir et créativité du travailleur social. Paris : Éditions ESF.

LEONARD, P. (1976). Towards a Paradigm for Radical Practice», dans R. Bailey et M. Brake, Radical Social Work. New York.

MALEWSKA-PEYRE, H. (1984). «Crise d'identité: problèmes de déviance chez les jeunes immigrés », Les temps modernes.

MALEWSKA-PEYRE, H. (1985). "Processus de socialisation des adolescents-enfants de migrants en situation interculturelle », dans C. Clavet, L'interculturel en éducation et en sciences humaines. Université de Toulouse - Le Mirail.

MALEWSKA-PEYRE, H. (1989). "Problèmes d'identité des adolescents-enfants de migrants et travail social », dans C. Camilleri et M. Cohen-Emerique, Chocs de cultures, op. cit.

MCPHEETERS, H. et R. RYAN (1971). A Core of Competence for Baccalaureat Social Welfare and Curriculum Implications. Atlanta: Southern Regional Education Board.

MOREAU, M. (1979). "A Structural Approach to Social Work», Revue canadienne d'éducation en service social, vol. 5, $\mathrm{n}^{\circ} 1$.

MOREAU, M. (1991). «L'infériorisation sociale: un jeu de simulation pour l'enseignement de l'empathie à l'égard des minorités opprimées », Service social, vol. 40, $\mathrm{n}^{\circ} 3$.

MURRY, G. (1975). «Remarques d'un sociologue à propos du service social», Connexions, 15. 
NALTChAyAN, M. et al. (1991). Esquisse de "grille» d'outil, pour l'intervenant confronté à une situ ation-problème en contexte interculturel. Ateliers pédagogiques, mai 1991. Centre de services sociaux du Montréal métropolitain (document miméographié).

PAuzé, R. et M. PetitClerC (1986). "LL'offre et la demande: le danger pour l'intervenant d'offrir plus qu'on ne lui demande ou qu'il n'est en mesure d'offrir», Systèmes humains, 2.

PeRLMAN, H.H. (1973). L'aide psychosociale interpersonnelle. Paris : Le Centurion.

PIGLER-CHRISTENSEN, C. (1986). «Cross-Cultural Social Work Practice: Fallacies, Fears and Failings ", Intervention, $\mathrm{n}^{\circ} 74$.

POISSON, P. (1988). "Travailler avec les familles non volontaires», Thérapie familiale, 4.

PRAY, L. (1947). Social Work in a Revolutionary Age. New York: Columbia University Press.

PRIEUR, B. (1989). «Aide demandée, aide imposée », Informations sociales, n 1.

SAYAD, A. (1989). «Éléments pour une sociologie de l'immigration», Les Cahiers internationaux de psychologie sociale.

SELVINI-PALAZZOLI, M. et al. (1984). "Le problème du référent en thérapie familiale», Thérapie familiale, 2.

SERRANO, J.A. et C. DIPP (1986). «La famille du migrant: adaptabilité et cohésion », Thérapie familiale, 1.

SERRANO, ).A. (1989). "La thérapie avec les familles des migrants », Thérapie famile, 4.

St-ARNAUD, Y, (1983). Devenir autonome. Montréal: Éditions Le Jour.

STERLIN, C. (1987). « La référence culturelle dans une pratique psychiatrique en milieu haïtien à Montréal », dans E. Corin et al., Regards anthropologiques en psychiatrie. Montréal : Éd. du GIRAME.

TILMANS OSTYN, E. (1985). "Analyse de l'enjeu de la "demande" au lieu de l'analyse de la plainte», Thérapie familiale, 3.

VAN CAMPENHOUT, L. (1989). «Le travailleur social : entre l'institution et l'usager», Revue d'action sociale, 6.

VASQUEZ, A. (1984). "Les implications idéologiques du concept d'acculturation », Cahiers de sociologie économique et culturelle, 1.

ZASTROW, C. et K.K. KIRST-ASHMAN (1987). Understanding Human Beha vior and the Social Environment. Chicago: Nelson-Hall Publishers. 